\title{
Non-Gaussian spectra in cosmic microwave background temperature anisotropies
}

\author{
Pedro G. Ferreira \\ Center for Particle Astrophysics, University of California, Berkeley, California 94720-7304 \\ João Magueijo \\ The Blackett Laboratory, Imperial College, Prince Consort Road, London SW7 2BZ, United Kingdom
}

(Received 16 October 1996)

\begin{abstract}
Gaussian cosmic microwave background skies are fully specified by the power spectrum. The conventional method of characterizing non-Gaussian skies is to evaluate higher order moments, the $n$-point functions, and their Fourier transforms. We argue that this method is inefficient, due to the redundancy of information existing in the complete set of moments. In this paper we propose a set of new statistics or non-Gaussian spectra to be extracted out of the angular distribution of the Fourier transform of the temperature anisotropies in the small field limit. These statistics complement the power spectrum and act as localization, shape, and connectedness statistics. They quantify the generic non-Gaussian structure, and may be used in more general imageprocessing tasks. We concentrate on a subset of these statistics and argue that while they carry no information in Gaussian theories, they may be the best arena for making predictions in some non-Gaussian theories. As examples of applications we consider superposed Gaussian and non-Gaussian signals, such as point sources in Gaussian theories or the realistic Kaiser-Stebbins effect. We show that in these theories non-Gaussianity is only present in a ring in Fourier space, which is best isolated in our formalism. Subtle but strongly nonGaussian theories are also written down for which only non-Gaussian spectra may reveal non-Gaussianity. [S0556-2821(97)04306-3]
\end{abstract}

PACS number(s): 98.80.Cq, 98.70.Vc, 98.80.Hw

\section{INTRODUCTION}

Gaussianity plays a central role in current theories of structure formation [1]. Inflationary theories are normally invoked to justify Gaussianity [2] but, historically, simplicity was perhaps what first motivated this assumption. As data have started to flood cosmology, however, the problem of testing Gaussianity has reappeared both in cosmic microwave background (CMB) analysis [3], and galaxy survey analysis [4]. A trend in data analysis has been established which relies on Gaussianity and a lingering feeling exists that the whole thing might fall through should the data prove to be non-Gaussian in the first place. Furthermore, structure formation theories exist which in one way or another predict non-Gaussian primordial fluctuations. Cosmic strings and textures [5] provide two such examples. Pinning down what precise non-Gaussian predictions such theories can make is a task crying for a comprehensive formalism for quantifying general non-Gaussianity. Finally, even if the "signal" is Gaussian, it may happen that a non-Gaussian noise component is present, e.g., unresolved point sources [6]. A precise prediction of their observational properties could then assist in their subtraction from data before the final theoretical analysis is performed.

One is, therefore, left with the problem of how to test Gaussianity, and how to quantitatively specify the most general non-Gaussian theory. Several tests for non-Gaussianity have been proposed in the past. Peaks' statistics [7,8], topological tests $[9,10]$, the three-point correlation function $[3,11]$, skewness and kurtosis $[12,13]$, and temperature and temperature gradient histograms [14] are the most topical examples. In some cases these tests were only shown to be applicable for rather artificial non-Gaussian distributions [9].
In other cases the tests were applied only to extremely nonGaussian signals, or the eroding effects of Gaussian noise were not explored [15].

These tests, however, are by no means exhaustive. One can always devise a non-Gaussian theory which evades detection by everyone of these tests, even when the hard realities of experiment do not fully erase signal non-Gaussianity. The only way to fully ascertain Gaussianity is to apply to data a comprehensive formalism for encoding nonGaussianity in its broadest generality. The $n$-point correlation function provides such a framework, and it has long been used in cosmology [16] and other branches of physics [17]. Computing the $n$-point function for large $n$ is, however, a practical impossibility. Taking the Cosmic Background Explorer (COBE) data as an example [3], only the three-point function has been computed, and even in that case attention was restricted to the pseudocollapsed and equilateral slices.

In Sec. II we start off by showing how the $n$-point correlation functions for $n$ up to any $N>3$ contain redundant information. For Gaussian fields all the $N>2$ correlators can be determined from the two-point correlator. We show that even for the most general non-Gaussian theory information encoded in the $N>3$ correlator is dependent on information in lower order correlators. Furthermore, we show that one can never be sure that by truncating the infinite correlator series at some $N$ one has all the information about the most general non-Gaussian theory. Strongly non-Gaussian theories may be written down which have Gaussian moments up to any given order $N$. The $n$-point function formalism then appears to have two drawbacks: redundancy and impractical complexity. We shall argue that these two drawbacks are due to each other, and that they may be eliminated altogether.

In Sec. III, we propose an alternative formalism for com- 
prehensively encoding non-Gaussianity. In the guise to be used in this paper the formalism lives naturally in Fourier space, and we have chosen to highlight non-Gaussianity other than that in the phases. The idea of looking for nonGaussianity in Fourier space has been disfavored in the past. It is argued that localized non-Gaussianity in real space (such as what is produced in cosmic string or texture scenarios) will be obscured in Fourier space due to the central limit theorem. It is also often assumed that a Gaussian field can be accurately modeled as the Fourier transform of a field whose randomness is solely in the phases. However, as we argue in Sec. II, looking in Fourier space allows us to probe the nonGaussian nature of the field at specific scales, a fact which is particularly useful when one can model the field as combination of a Gaussian field which dominates on certain scales and a non-Gaussian field which dominates on others. Another very strong reason for considering Fourier space statistics seriously is the fact that the highest resolution measurements of CMB anisotropies will be performed by interferometric devices, which naturally measure quantities in Fourier space (the " $u v$ plane").

Therefore, ignoring prejudice, in Sec. III we define a set of "non-Gaussian spectra" in terms of the Fourier transform of the temperature anisotropies. Our definitions follow up the proposals in [18], but they are substantially more practical. We then characterize the probability distribution function of these spectra in Gaussian theories and in Appendix B give a physical interpretation of the qualities which they measure. We set these quantities up so that while they contain all the information degrees of freedom, they do away with any redundancy. As a result, we come up with a formalism which shares with the $n$-point correlators the property of being comprehensive, but with the advantage that it is computable and nonredundant. Within the large set of statistics considered in this paper we concentrate on a set of statistics which only use the information in the absolute value of the Fourier modes. These are grouped in two types of spectra: the ring spectrum and the inter-ring spectrum. For the sake of maximal originality we leave to a future publication the investigation of the role played by the more prosaic phase information.

In Sec. IV we consider three different applications. First, we consider the case of a point source which is obscured by Gaussian fluctuations. Second, we consider the realistic temperature anisotropy induced by a cosmic string, including both the post-recombination Kaiser-Stebbins effect and the Gaussian fluctuations at the surface of last scattering. Finally, we construct a strongly non-Gaussian theory, a theory which produces skies which have a zero probability of occurring in a Gaussian theory. To all these examples we apply a battery of conventional statistics and show that they evade any detection of non-Gaussianity. We show, however, that our statistics reveal the non-Gaussian nature of the skies.

In Sec. V we conclude by discussing the limitations of these statistics and their possible extensions.

\section{THE $\boldsymbol{n}$-POINT CORRELATION FUNCTION}

We start by reviewing the $n$-point correlation function formalism. We then introduce the concept of $u v$-plane invariants, that is quantities which are made up of Fourier modes $a(\mathbf{k})$, and which are invariant under rotations and translations. We show how the Fourier transform of the $n$-point correlation function is made up of $u v$-plane multilinear invariants. One may then count the number of degrees of freedom in the Fourier modes for a given sky coverage. By doing so we show that the $n$-point correlators for $n$ up to a certain $N$ contain information which can only be redundant. This will set the tone for the next section: trying to do away with the redundancy and complexity of the $n$-point correlation function.

\section{A. The $n$-point correlation function and its transform}

We consider $\mathrm{CMB}$ data in the small angle limit, when projecting onto a planar patch is suitable. Since data may come in either real or Fourier space, we hope to address the problem of non-Gaussianity in terms of these two descriptions. In this paper, however, we will concentrate on the Fourier space description, and thus produce statistics better suited to interferometers. We shall use the convention

$$
\frac{\Delta T(\mathbf{x})}{T}=\int \frac{d \mathbf{k}}{2 \pi} a(\mathbf{k}) e^{i \mathbf{k} \cdot \mathbf{x}}
$$

The $n$-point correlation function is defined as the expectation value of the product of any $n$ temperatures. Translational and rotational invariance make redundant the position of one of the points and the direction of another. Hence, the $n$-point function may be written as a function of $\left(x_{2}, \mathbf{x}_{3}, \ldots, \mathbf{x}_{n}\right)$ in the form

$$
C^{n}\left(x_{2}, \mathbf{x}_{3}, \ldots, \mathbf{x}_{n}\right)=\left\langle\frac{\Delta T\left(\mathbf{x}_{1}\right)}{T} \ldots \frac{\Delta T\left(\mathbf{x}_{n}\right)}{T}\right\rangle .
$$

The two-point correlation function and its Fourier transform, the angular power spectrum $C(k)$ are well known. They fully specify Gaussian fluctuations. For Gaussian fluctuations nonvanishing higher order correlation functions exist, but they are redundant as they can be obtained from the two-point correlation function. This is not the case in non-Gaussian theories, for which the $n$-point correlators act not only as a non-Gaussianity indicator, but are also an indispensable fluctuation qualifier, as much as the power spectrum.

The angular power spectrum may be generalized for $n>2$ by Fourier analyzing the $n$-point function

$$
\begin{aligned}
& C^{n}\left(x_{2}, \mathbf{x}_{3}, \ldots, \mathbf{x}_{n}\right) \\
& \quad=\int \frac{d k_{2}}{(2 \pi)^{1 / 2}} \cdots \frac{d \mathbf{k}_{n}}{2 \pi} C^{n}\left(k_{2}, \ldots, \mathbf{k}_{n}\right) e^{i k_{2} x_{2}} \ldots e^{i \mathbf{k}_{n} \cdot \mathbf{x}_{n}} .
\end{aligned}
$$

In general, $C(k)$ is more predictive than $C(x)$, as it tells us how much power exists on a given scale. In the same way one may expect the transform $C^{n}\left(k_{2}, \ldots, \mathbf{k}_{n}\right)$ to be more predictive than its configuration space counterpart, as it tells us how much non-Gaussianity exists on each scale. We shall call $C^{n}\left(k_{2}, \ldots, \mathbf{k}_{n}\right)$ a non-Gaussian spectrum. One may also define Gaussian spectra as correlators of the $a(\mathbf{k})$ modes:

$$
\left\langle a\left(\mathbf{k}_{1}\right) \cdots a\left(\mathbf{k}_{\mathbf{n}}\right)\right\rangle=\delta\left(\mathbf{k}_{1}+\cdots \mathbf{k}_{\mathbf{n}}\right) C^{n}\left(k_{2}, \ldots, \mathbf{k}_{n}\right),
$$


where the $\delta$ function and functional form of $C^{n}$ result from the requirements of translational and rotational invariance [see Eq. (5) below]. Using Eq. (1), one may easily check that the two definitions (3) and (4) of $C^{n}\left(k_{2}, \ldots, \mathbf{k}_{n}\right)$ agree.

Non-Gaussian spectra are more complicated than power spectra, since they are functions of many variables. As $n$ increases, one is left with the problem of how to pack so much information. We will, however, show that most of the information encoded in $C^{n}\left(k_{2}, \ldots, \mathbf{k}_{n}\right)$ is largely redundant, even for the most general non-Gaussian fluctuation.

\section{B. $u v$-plane multilinear invariants as components of the $\boldsymbol{n}$-point correlation function}

Here, we show an equivalent route to non-Gaussian spectra. This route draws on work in [18], where the spherical harmonic coefficients $a_{m}$ are used to define quantities other than the $C_{\ell}$ spectrum which are invariant under the threedimensional (3D) rotation group. $m$ spectra and inter- $\ell$ correlators appear as supplementary information. These spectra are multilinear combinations of the $a_{m}^{l}$ which can be generally written as sums of products of Clebsch-Gordan coefficients. It can be shown that they act as a decomposition of the $n$-point function on the sphere in a suitable base made up of Legendre polynomials and spherical harmonics. These spectra are trivial to implement on a computer, but are formally quite complicated for large $\ell$. An explicit expression for the quadrupole $m$ shape was given in [18] with a suggested application to texture scenarios. Fortunately, at very high $\ell$ one may simply reformulate the problem in terms of the Fourier representation of small patches. $m$ spectra and inter- $\ell$ correlators then become very simple. They reappear as $u v$-plane invariants, that is quantities made up of the $a(\mathbf{k})$ modes, and which are invariant under $2 \mathrm{D}$ rotations and translations (the projected 3D rotation group).

The non-Gaussian spectra $C^{n}\left(k_{2}, \ldots, \mathbf{k}_{n}\right)$ are invariant under rotations and translations. This requirement may also be imposed on any set of qualifiers of a random field which statistically satisfies these invariances. Under a rotation $R_{\theta}$ and a translation along a vector $\mathbf{t}$ the Fourier components transform as in

$$
R_{\theta}(a(\mathbf{k}))=a\left(R_{\theta}(\mathbf{k})\right), \quad T_{\mathbf{t}} a(\mathbf{k})=e^{i \mathbf{k} \cdot \mathbf{t}} a(\mathbf{k}) .
$$

A systematic way to generate invariants out of the $a(\mathbf{k})$ is to consider multilinear combinations, that is sums of products of $n$ modes $a(\mathbf{k})$ (monomials). For these to be invariant under translations it is necessary that the vectors $\mathbf{k}_{i}$ used in each monomial add up to zero. To achieve invariance under rotations one must then, for each monomial, average over all possible rotations of the $\mathbf{k}_{i}$ configuration used. One may formally write the most general multilinear invariant of order $n$ as

$$
I^{(n)}=\frac{1}{N_{\theta}} \sum_{\theta} \prod_{i=1}^{n} a\left(\mathbf{k}_{i}\right)
$$

in which the vectors $\mathbf{k}_{i}$ considered in each product must add up to zero and always take the same configuration, and $N_{\theta}$ is the total number of possible rotations of the configuration, should Fourier space be discretized. For $n=2$ the only in-

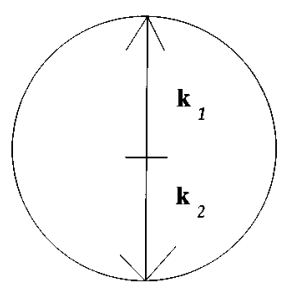

$n=2$ $n=3$

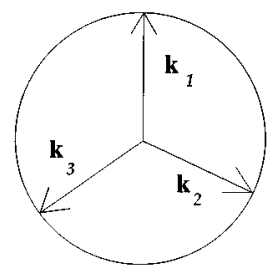

FIG. 1. The most general second order invariant (left) is the angular power spectrum, obtained by multiplying the mode $\mathbf{k}_{1}$ amplitude with the mode $\mathbf{k}_{2}=-\mathbf{k}_{1}$ amplitude, and averaging over directions. The result can only depend on $k$. On the right we show one possible configuration giving a third order invariant, the one where all three vectors have the same moduli. Then, for each $k$ all three vectors are determined from the requirement that they must add up to zero. Averaging over directions produces an invariant.

variant for each $k$ is the angular power spectrum. Given a vector $\mathbf{k}$, the requirement that the second vector in the binomial adds to zero fully determines the second vector. Averaging over all rotations makes the direction of the first vector irrelevant. The invariant (6) then reduces to

$$
I^{(2)}(k)=\frac{1}{N} \sum_{|\mathbf{k}|=k}|a(\mathbf{k})|^{2} .
$$

For the third order invariants one now has an invariant which depends on a vector and a scalar. Independent invariants are parametrized by the third vector and the relative direction of the second vector. The first vector is fully determined by the requirement that the three vectors add to zero. The actual directions of the second and third vectors are made redundant by taking the circular average. A particularly interesting third order invariant may be obtained if one demands that the three vectors used all have the same moduli. Then, for each $k$ only one invariant exists, the one obtained with the configuration plotted in Fig. 1.

Diagrammatically, one may then write down the most general invariant for any order, rapidly bumping into unwanted proliferation. The procedure, however, is very simple, and reduces to Eq. (6) and the various independent diagrams it allows. The most general multinomial invariant of degree $n$ is a function of $\left(k_{2}, \mathbf{k}_{3}, \ldots, \mathbf{k}_{n}\right)$. Hence, the non-Gaussian spectra defined in the decomposition of the $n$-point correlation function correspond to the most general multinomial invariant one may construct out of the $a(\mathbf{k})$.

\section{Exposing the redundancy of the $\boldsymbol{n}$-point function}

The approach just devised has the advantage of allowing us to expose the redundancy of the $n$-point correlation function. Let us start by counting the number of degrees of freedom present in the Fourier modes produced by a given measurement. If we had full sky coverage then there would be $2 k+1$ modes per unit of $k$. Finite sky coverage has the effect of correlating neighboring modes among these, thereby reducing the number of independent modes per unit of $k$ to $2 k f_{\text {sky }}$, if $f_{\text {sky }}$ is the fraction of sky covered. An alternative Fourier space discretization is then required, so that the modes in the new mesh are quasi-uncorrelated, while encod- 
ing all the statistical information in the original modes. This may be done with a so-called uncorrelated mesh (see [19]). There is some arbitrariness in where the new mesh is laid. This arbitrariness allows us to be sloppy with the invariances imposed in the previous section, since any vector $\mathbf{k}$ may now be placed anywhere in the uncorrelated mesh cell. Hence, the angles required by configurations such as the ones in Fig. 1 should be seen as flexible, as far as the mesh resolution in concerned.

Let us now consider a generic $\Delta k=1$ ring containing $N_{\text {ring }} \approx 2 k f_{\text {sky }}$ uncorrelated mesh points. Since there are three degrees of freedom in rotations and translations one may not build more than $N_{\text {ring }}-3$ independent invariants per unit of $k$, plus three invariants relating adjacent $\Delta k=1$ rings. The number of multilinear invariants making up the $n$-point function transform is vastly larger. Even if we restrict ourselves to invariants made up only of modes in each ring, the number of invariants is 1 for $n=2,3$ (see Fig. 1), then, for $n>3$, of order $O\left(N_{\text {ring }}^{n-3}\right)$, if $N_{\text {ring }} \gg 1$.

The situation gets worse if we consider inter-ring multinomial invariants. Let us now consider a square in Fourier space with $N_{p} \times N_{p}$ uncorrelated mesh points. Then, for large $N_{p}$ the number of multilinear invariants of order $n$ in all rings is of order $O\left(N_{p}^{n-1}\right)$. The number of independent mesh points, on the contrary, is of order $O\left(N_{p}^{2}\right)$.

Hence, there must be an algebraic dependence between all the multilinear invariants. The information encoded in the higher order correlators must, therefore, repeat itself in any theory, Gaussian or not. We, therefore, argue that the $n$-point function formalism, while comprehensive, is not systematic. This is not to say that some truncation of the correlator series might not be useful as a non-Gaussianity test. In particular, we feel that ring multinomial invariants, such as the cubic one depicted in Fig. 1, may be useful nonGaussianity tests.

\section{RING AND INTER-RING SPECTRA}

We now propose an alternative packaging for the information in Fourier space. Comparing it with the $n$-point transform, it is simpler, does away with redundancy, and has an immediate physical interpretation. We divide the $u v$ plane in $\Delta k=1$ rings where $N_{k}=2 k f_{\text {sky }}$ independent modes lie. Out of these we may build $N_{k}-3$ invariants. In whatever we do we shall always make sure that the formalism proposed produces the power spectrum $C(k)$ as the first of these quantities. The other $C(k, m)$, for $m=1, \ldots, N_{k}-4$, are the ring spectra. We shall not consider multilinear invariants, but shall search for alternative prescriptions. On top of these, for each two adjacent rings there will be three invariants, the inter-ring correlators. Given the arbitrariness of the Fourier mode mesh exact positions we may also be justified in building simply $N_{k}$ noninvariant quantities for each ring, as long as we know how they transform. We found the latter attitude more practical, but shall give in Appendix A the correct prescription for building properly invariant quantities.

For a Gaussian theory the probability of a given map depends only on the map power spectrum. Consider then a very non-Gaussian map by which we mean something we visually recognize as very structured. Consider also various other maps with the same power spectrum, but which we visually recognize as very Gaussian. All these maps, Gaussian looking or not, have the same probability in Gaussian theories. In non-Gaussian theories, on the contrary, the probability of a given map depends on more than its power spectrum. Hence, within the set of maps considered above, it may happen that the non-Gaussian-looking map is now considerably more probable than the other maps. The point we wish to make is that non-Gaussianity arises not from structured maps being less likely in Gaussian theories, but from structured maps being more likely in non-Gaussian theories.

This seemingly innocent remark has two important implications. First, it implies that the natural variables for nonGaussianity spectra should be uniformly distributed in Gaussian theories. In contrast, at least in some non-Gaussian theories, the same variables should have peaked distributions. Hence, non-Gaussian spectra should carry no information whatsoever in Gaussian theories, but they should be highly predictive at least in some non-Gaussian theories.

A second implication is that disproving Gaussianity on its own merits is a contradiction in terms. One can always disprove a given non-Gaussian theory on its own merits by measuring a non-Gaussian spectrum and finding it to be away from the theoretically predicted ridge. However, any non-Gaussian spectrum measurement is equally probable in Gaussian theories, and so it can never be used as an evidence against Gaussianity. Disproving Gaussianity is then a matter dependent on the available competing non-Gaussian theories. If one measures a non-Gaussian spectrum spot on the prediction of a well motivated non-Gaussian theory then this is a strong evidence in favor of that non-Gaussian theory. One may simply argue that the non-Gaussian theory has predicted the observation with much larger probability than the Gaussian theory. Pedantically, the observation has not disproved Gaussianity. However, it has discredited Gaussianity massively in the face of the more predictive competing nonGaussian theory.

It is under the requirement that non-Gaussian spectra ought to be uniformly distributed in Gaussian theories that we now proceed to define non-Gaussian spectra. Consider a ring of the $u v$ plane where $N_{k}$ independent complex modes $a\left(\mathbf{k}_{i}\right)=\operatorname{Re}\left[a\left(\mathbf{k}_{i}\right)\right]+i \operatorname{Im}\left[a\left(\mathbf{k}_{i}\right)\right]$ live. In Gaussian theories these are distributed as

$$
\begin{aligned}
F\left(\operatorname{Re}\left[a\left(\mathbf{k}_{i}\right)\right], \operatorname{Im}\left[a\left(\mathbf{k}_{i}\right)\right]\right) & \\
= & \frac{1}{\left(2 \pi \sigma^{2}\right)^{N_{k} / 2}} \exp \left[-\left(\frac { 1 } { 2 \sigma _ { k } ^ { 2 } } \sum _ { i = 1 } ^ { m _ { k } } \left\{\operatorname{Re}^{2}\left[a\left(\mathbf{k}_{i}\right)\right]\right.\right.\right. \\
& \left.\left.\left.+\operatorname{Im}^{2}\left[a\left(\mathbf{k}_{i}\right)\right]\right\}\right)\right],
\end{aligned}
$$

where $m_{k}=N_{k} / 2$. First, separate the $N_{k}$ complex modes into $m_{k}$ moduli $\rho_{i}$ and $m_{k}$ phases $\phi_{i}$ :

$$
\operatorname{Re}\left[a\left(\mathbf{k}_{i}\right)\right]=\rho_{i} \cos \phi_{i}, \quad \operatorname{Im}\left[a\left(\mathbf{k}_{i}\right)\right]=\rho_{i} \sin \phi_{i} .
$$

The Jacobian of this transformation is

$$
\left|\frac{\partial\left(\operatorname{Re}\left[a\left(\mathbf{k}_{i}\right)\right], \operatorname{Im}\left[a\left(\mathbf{k}_{i}\right)\right]\right)}{\partial\left(\rho_{i}, \phi_{i}\right)}\right|=\prod_{i=1}^{m_{k}} \rho_{i} .
$$


The $\left\{\rho_{i}\right\}$ may be seen as Cartesian coordinates which we transform into polar coordinates. These consist of a radius $r$ plus $m_{k}-1$ angles $\widetilde{\theta}_{i}$ given by

$$
\rho_{i}=r \cos \widetilde{\theta}_{i}^{i-1} \prod_{j=0}^{i n} \sin \widetilde{\theta}_{j}
$$

with $\sin \widetilde{\theta}_{0}=\cos \widetilde{\theta}_{m_{k}}=1$. In terms of these variables the radius is related to the angular power spectrum by $C(k)=r^{2} /\left(2 m_{k}\right)$. In general, the first $m_{k}-2$ angles $\tilde{\theta}_{i}$ vary between 0 and $\pi$ and the last angle varies between 0 and $2 \pi$. However, because all $\rho_{i}$ are positive all angles are in $(0, \pi / 2)$. The Jacobian of this transformation is

$$
\left|\frac{\partial\left(\rho_{1}, \ldots, \rho_{m_{k}}\right)}{\partial\left(r, \widetilde{\theta}_{1}, \ldots, \widetilde{\theta}_{m_{k}-1}\right.}\right|=r^{m_{k}-1} \prod_{i=2}^{m_{k}-1} \sin ^{m_{k}-i} \widetilde{\theta}_{i-1} .
$$

Polar coordinates in $m_{k}$ dimensions may be understood as the iteration of the rules

$$
\rho_{i}=r_{i} \cos \widetilde{\theta}_{i}, \quad r_{i-1}=r_{i} \sin \widetilde{\theta}_{i},
$$

in which $r_{i}$ is the radius of the shade $\left(m_{k}-i+1\right)$-dimensional sphere obtained by keeping fixed all $\rho_{j}$ for $j=1, \ldots, i-1$ :

$$
r_{i}=\sqrt{\rho_{i}^{2}+\rho_{i+1}^{2}+\cdots+\rho_{m_{k}}^{2}} .
$$

One may easily see that this is how 3D polars work, and also that the transform (11) follows this rule. Hence, one may invert the transform (11) with

$$
\widetilde{\theta}_{i}=\arccos \frac{\rho_{i}}{\sqrt{\rho_{i}^{2}+\rho_{i+1}^{2}+\cdots+\rho_{m_{k}}^{2}}}
$$

for $i=1, \ldots, m_{k}-1$.

The total Jacobian of the transformation from $\left\{\operatorname{Re}\left[a\left(\mathbf{k}_{i}\right)\right], \operatorname{Im}\left[a\left(\mathbf{k}_{i}\right)\right]\right\}$ to $\left\{r, \widetilde{\theta}_{i}, \phi_{i}\right\}$ is just the product of Eqs. (10) and (12). Hence, for a Gaussian theory one has the distribution

$$
\begin{aligned}
F\left(r, \widetilde{\theta}_{i}, \phi_{i}\right)= & \frac{r^{N_{k}-1} \exp \left[-\left(\frac{r^{2}}{2 \sigma_{k}^{2}}\right)\right]}{\left(2 \pi \sigma^{2}\right)^{N_{k} / 2}} \\
& \times \prod_{i=1}^{m_{k}-1} \cos \widetilde{\theta}_{i}\left(\sin \widetilde{\theta}_{i}\right)^{N_{k}-2 i-1} .
\end{aligned}
$$

In order to define $\widetilde{\theta}_{i}$ variables which are uniformly distributed in Gaussian theories, one may finally perform the transformation on each $\widetilde{\theta}_{i}$ :

$$
\theta_{i}=\sin ^{N_{k}-2 i}\left(\widetilde{\theta}_{i}\right)
$$

so that for Gaussian theories one has

$$
F\left(r, \theta_{i}, \phi_{i}\right)=\frac{r^{N_{k}-1} e^{-r^{2} /\left(2 \sigma_{k}^{2}\right)}}{2^{m_{k}-1}\left(m_{k}-1\right) ! \sigma_{k}^{N_{k}}} \times 1 \times \prod_{i=1}^{m_{k}} \frac{1}{2 \pi} .
$$

The factorization chosen shows that all new variables are independent random variables for Gaussian theories. $r$ has a $\chi_{N_{k}}^{2}$ distribution, the "shape" variables $\theta_{i}$ are uniformly distributed in $(0,1)$, and the phases $\phi_{i}$ are uniformly distributed in $(0,2 \pi)$.

The variables $\theta_{i}$ define a non-Gaussian shape spectrum, the ring spectrum. They may be computed from ring moduli $\rho_{i}$ simply by

$$
\theta_{i}=\left(\frac{\rho_{i+1}^{2}+\cdots+\rho_{m_{k}}^{2}}{\rho_{i}^{2}+\cdots+\rho_{m_{k}}^{2}}\right)^{m_{k}-i} .
$$

They describe how shapeful the perturbations are. If the perturbations are stringy then the maximal moduli will be much larger than the minimal moduli. If the perturbations are circular, then all moduli will be roughly the same. This favors some combinations of angles, which are otherwise uniformly distributed. In general, any shapeful picture defines a line on the ring spectrum $\theta_{i}$. A non-Gaussian theory ought to define a set of probable smooth ring spectra peaking along a ridge of typical shapes.

We can now construct an invariant for each adjacent pair of rings, solely out of the moduli. If we order the $\rho_{i}$ for each ring, we can identify the maximum moduli. Each of these moduli will have a specific direction in Fourier space; let $\mathbf{k}_{\max }$ and $\mathbf{k}_{\max }^{\prime}$ be the directions where the maximal moduli are achieved. The angle

$$
\psi\left(k, k^{\prime}\right)=\frac{1}{\pi} \operatorname{ang}\left(\mathbf{k}_{\max }, \mathbf{k}_{\text {max }}^{\prime}\right)
$$

will then produce an inter-ring correlator for the moduli, the inter-ring spectra. This is uniformly distributed in Gaussian theories in $(-1,1)$. It gives us information on how connected the distribution of power is between the different scales.

We have, therefore, defined a transformation from the original modes into a set of variables $\{r, \theta, \phi, \psi\}$. The nonGaussian spectra thus defined have a particularly simple distribution for Gaussian theories. They also comply with the uniformity requirement we have placed on non-Gaussian spectra in the discussion at the start of this section. We shall call perturbations, for which the phases are not uniformly distributed, localized perturbations. This is because if perturbations are made up of lumps statistically distributed but with well-defined positions, then the phases will appear highly correlated. We shall call perturbations, for which the ring spectra are not uniformly distributed, shapeful perturbations. We will identify later the combinations of angles which measure stringy or spherical shape of the perturbations. This distinction is interesting as it is, in principle, possible for fluctuations to be localized but shapeless, or more surprisingly, to be shapeful but not localized. Finally, we shall call perturbations, for which the inter-ring spectra are not uniformly distributed, connected perturbations. This turns out to be one of the key features of stringy perturbations. These three definitions allow us to consider structure in various layers. White noise is the most structureless type of perturbation. Gaussian fluctuations allow for modulation, that is a nontrivial power spectrum $C(k)$, but their structure stops there. Shape, localization, and connectedness constitute 

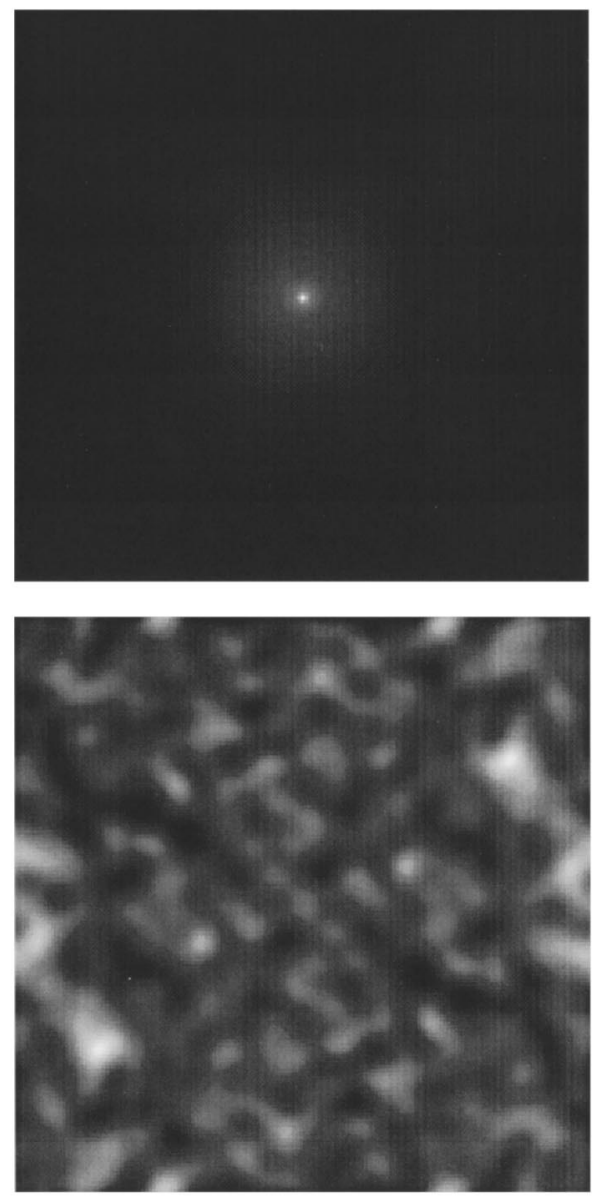

FIG. 2. The non-Gaussian signal (top) and the full signal, with the Gaussian superposition (bottom) for $\alpha=3, k_{c}=0.1$, and $k_{g}=5$. In the map on the top the skewness is 2.9 and the kurtosis 11.4. In the map on the bottom the skewness is -0.02 and the kurtosis -0.38 .

the three next levels of structure one might add on. Standard visual structure is contained within these definitions, but they allow for more abstract levels of structure. We will show in Appendix B what these concepts mean with reference to visual structure.

In the formulation above there is a minor flaw which we found inconsequent, given the practical advantages gained. This flaw is spelled out and corrected in Appendix A, but we have chosen not to do so in the main body of this paper. In Appendix A we also mention what can be done with the phases $\phi$. This is, however, outside the scope of this paper, where we have decided to investigate the practical applications of the less investigated ring and inter-ring spectra.

\section{APPLICATIONS}

Historically, much attention has been paid to the nonGaussianity in the phases $\phi$. As mentioned above, it has frequently been assumed that the prescription of random phases in Fourier space leads to Gaussian perturbations. Evidence of peculiar behavior of the phases was shown in numerical simulations of $\mathrm{CMB}$ anisotropies from cosmic strings $[24,23]$. Little attention has been given to the $\rho$ 's. In the following three applications we will focus on the statis-

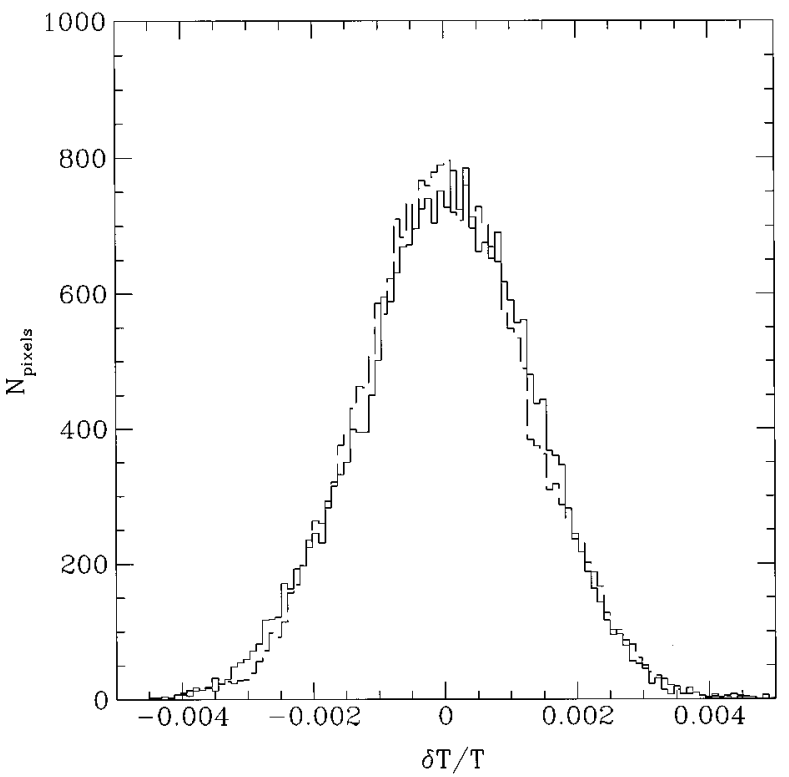

FIG. 3. Histograms of the temperature distributions for the nonGaussian map (solid line) and purely Gaussian map with the same power spectrum (dashed line).

tics only involving the $\rho$ 's and show that, in these cases, they are good non-Gaussian indicators.

In all these examples we will consider maps with $160^{2}$ pixels with no noise; it has been customary to apply the various standard statistics to the raw non-Gaussian signal superposed with small scale Gaussian noise, but no attempt has been made at studying the effects of large scale Gaussian fluctuations. As we will argue there are physically motivated reasons for doing so. With the intent of keeping the different effects separate we will analyze this latter case. The addition of noise should be studied when considering specific observation strategies.

We will quote all values of the wave number $k$, using uncorrelated mesh units, i.e., following the discussion of Sec. II, we will start labeling the wave numbers in unit intervals, from the smallest up to the largest. The width of the rings are, therefore, $\Delta k=1$.

\section{A. Unresolved point source on a Gaussian signal}

As a first application of these statistics, let us consider a Gaussian signal when non-Gaussian foregrounds are present. We know that this is the case in real CMB measurements and there exist a series of techniques which allow one to separate the two signals, using a combination of spectral and spatial information. A more difficult situation occurs when one considers unresolved point sources. In this case, either one uses additional information about the patch of the sky one is observing [6] or one has to make assumptions and the best one can achieve is to subtract them on a statistical basis.

Let us consider a simple case which illustrates the weakness of current methods for checking non-Gaussianity but highlights the strengths of our technique. Suppose that the field is sufficiently small for only a small number of point sources to be present. Also, suppose that the signal is Gaussian and that it has reached the Silk damping tail. The probed spectrum will then go to white noise at the scale of the field 


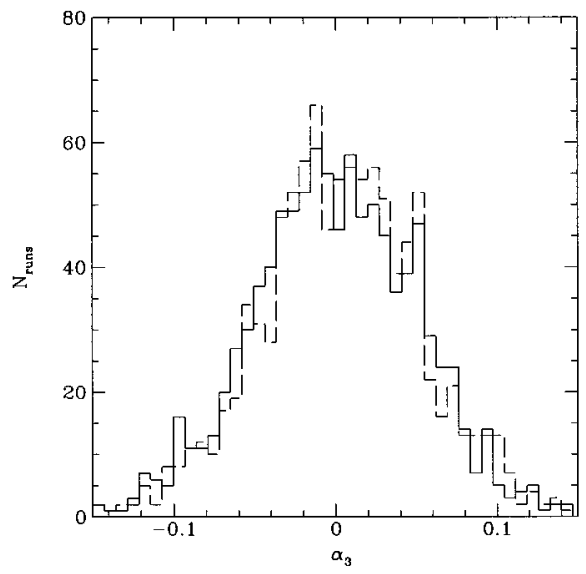

size, but converge to the raw spectrum otherwise [19]. A fitting formula for the power spectrum of the Gaussian signal is

$$
P_{g}(k)=\alpha \exp \left(\frac{-k^{2}}{2 k_{g}^{2}}\right) .
$$

On top of this, one must either firmly believe that the signal is Gaussian, or that the signal is non-Gaussian, but of a distinctively different shape. Now, let a single unresolved source be present in the field. Let the source be perfectly circular, and have a Fourier space falloff of the form

$$
P_{n g}(k)=\frac{1}{1+\left(k / k_{c}\right)^{4}} \text {. }
$$

The phases are all correlated and arranged so as to center the configuration and the angles $\theta$ correspond to a perfectly circular configuration. All moduli are exactly equal the square root of the power spectrum. This is a shapeful, localized, and connected perturbation, visually recognizable as highly nonGaussian (see Fig. 2). Although we are using it as a toy model for an unresolved source, this is inspired by a spot produced by a texture undergoing perfect, spherically symmetric collapse.

In Fig. 2 we show the point source, and the signal mixed with the point source for the case $\alpha=3, k_{c}=0.1$, and $k_{g}=5$. What has started as visually very non-Gaussian disappears completely with the addition of a Gaussian signal. A real space subtraction of the source is bound to fail. From inspecting the histogram of temperatures at each realization one finds that, comparing with a purely Gaussian map with the same overall power spectrum, they look the same (see Fig. 3). A more thorough analysis would lead us to calculate the skewness $\alpha_{3}$ and kurtosis $\alpha_{4}$ of the maps:

$$
\alpha_{3}=C^{3}(0,0) /\left[C^{2}(0)\right]^{3 / 2}, \quad \alpha_{4}=\left\{C^{4}(0,0,0) /\left[C^{2}(0)\right]^{2}\right\}-3,
$$

or better yet, estimate the distribution of $\alpha_{3}$ and $\alpha_{4}$. In Fig. 4 we superpose histograms of of skewness (left panel) and kurtosis (right panel) for the non-Gaussian theory and for the purely Gaussian theory; clearly, the Gaussian behavior on large scales is dominating the effect of the point source.

One useful statistic to apply is the accumulated density of peaks above a given threshold. It was shown in [7] that, for a
Gaussian field, the density of peaks over a threshold $\mu \sigma$, where $\sigma=\sqrt{\left\langle|\delta T / T|^{2}\right\rangle}$, is approximately given by

$$
\begin{aligned}
N_{\text {peaks }}(\mu)= & \frac{1}{4 \pi \sqrt{3} \theta_{*}^{2}} \max \left[1,\left(\frac{6}{\pi}\right)^{1 / 2} \gamma^{2} \mu \exp \left(-\mu^{2} / 2\right)\right. \\
& \left.+\operatorname{erfc}\left\{\frac{\mu}{\left[2\left(1-2 \gamma^{2} / 3\right)\right]^{1 / 2}}\right\}\right],
\end{aligned}
$$

where $\gamma$ and $\theta_{*}$ are dimensionless ratios of the first three moments of the random field. We can apply this statistic to our maps, and in Fig. 5 we compare the peak density of the non-Gaussian maps with that of the pure Gaussian theory (with the same power spectrum). Although there is a slight difference for low (negative thresholds), the two peak densities are essentially indistinguishable.

We can now apply the approach we have devised. The non-Gaussianity will only become evident on small scales, i.e., for large $k$ 's in the Fourier plane. In fact, we can find an analytical expression for the ring spectrum of a perfectly

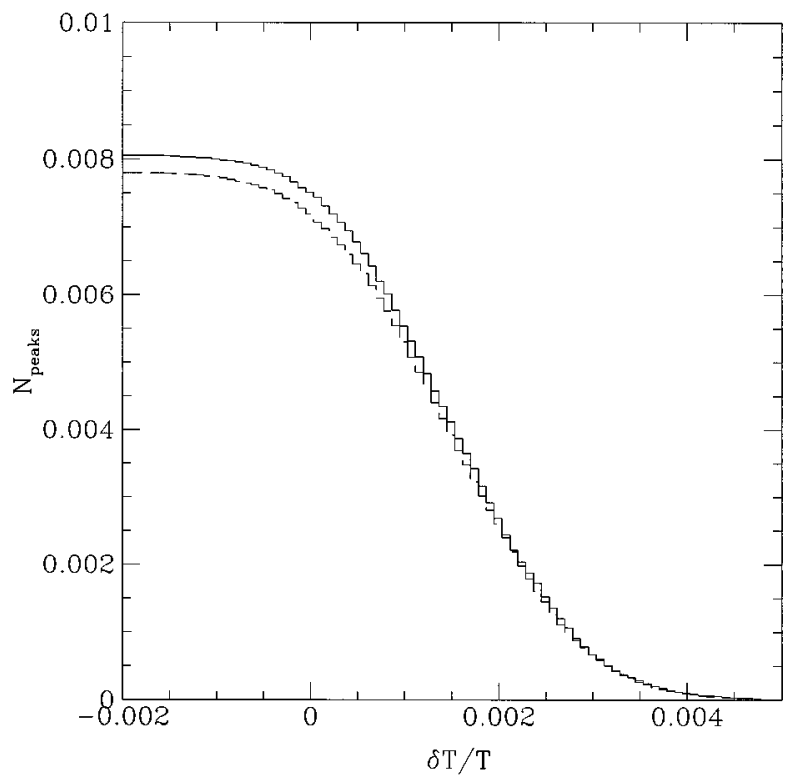

FIG. 5. The density of peaks above a threshold $\delta T / T$ for the non-Gaussian theory (solid line) and the purely Gaussian theory (dashed line). The curves are averaged over 20 runs. 


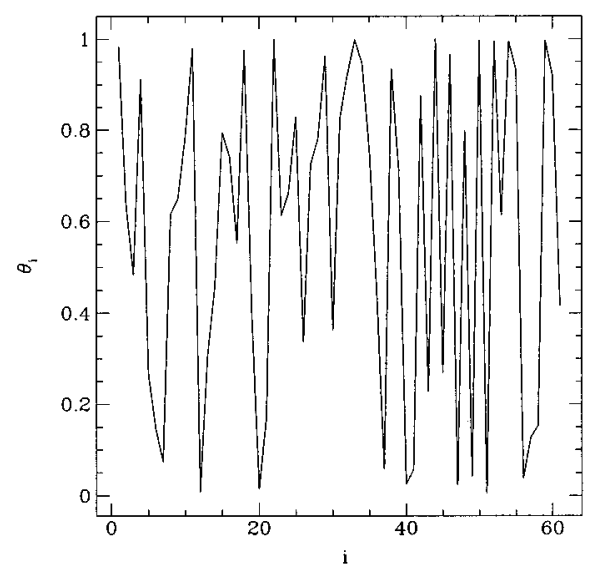

circular configuration: all moduli are equal to the same value $\rho_{i}=I$. Then, the ring spectrum is

$$
\theta_{i}^{\mathrm{circ}}=\left(\frac{m_{k}-i}{m_{k}-i+1}\right)^{m_{k}-i}
$$

For large values of $m_{k}$ this ring spectrum is approximately $1 / e$ for all $i$, until $i$ approaches $m_{k}-1$, where the spectrum rises to $1 / 2$. As shown in Fig. 6 (left panel), the ring spectrum at a low $k$ is indeed consistent with a uniform distribution (the $\theta_{i}$ 's are uniformly distributed between 0 and 1). As $k$ increases the angles $\theta_{i}$ start accumulating around the circular ridge. Soon, the point source dominates the signal, a fact evidenced by a perfectly circular ring spectrum. Well into the non-Gaussian region of Fourier space (where the Gaussian signal is strongly suppressed), we find a clean signal as shown in Fig. 6 (right panel).

This example illustrates the main idea and the main weakness behind our technique. The main idea consists of trying to identify the particular scale on which non-Gaussianity is evident and, clearly, this is best done in Fourier space. In this case (with no experimental small scale noise), one simply needs to look at $k$ 's on sufficiently small scales; the inclusion of Gaussian noise would introduce an outer limit in Fourier space, reducing the region of non-Gaussianity to a finite ring.

As for the main weakness we point out that the shape spectrum $\theta_{i}$ is sensitive only to the global shape of the map. While one point source leads to a very clean distribution of power around rings in Fourier space, if one has more than a few point sources then this will become less clear. Although for a set of $N$ sources one will have a very distinct signal (a smooth line as opposed to a random distribution of $\theta_{i}$ ), it becomes more difficult to distinguish the sources on a firm basis from a purely Gaussian signal. This leads us to establish the best operational strategy for this method to work: choose small fields and analyze them separately. In doing this one will be probing the scales on which non-Gaussianity becomes dominant with less objects to pick out. The fact that interferometric measurements of the $\mathrm{CMB}$ are constrained to small fields leads us to believe this to be a sensible prescription for $u v$-plane data analysis. Recent experience with such measurements [6] seems to indicate that indeed in each field there are only a few problematic sources (maybe one or two).
FIG. 6. The ring spectra for two rings $k=20$ and $k=50$.
One of the best motivated theories of non-Gaussian structure formation is that of cosmic strings. Following a primordial phase transition, linelike concentrations of energy could form in certain grand unified theories [5]. This network of strings would then evolve into a self-similar scaling regime, perturbing matter and radiation during its evolution. The nonlinear evolution of the strings should lead to a nonGaussian distribution of fluctuations; more specifically, the effect of strings on radiation after recombination should lead
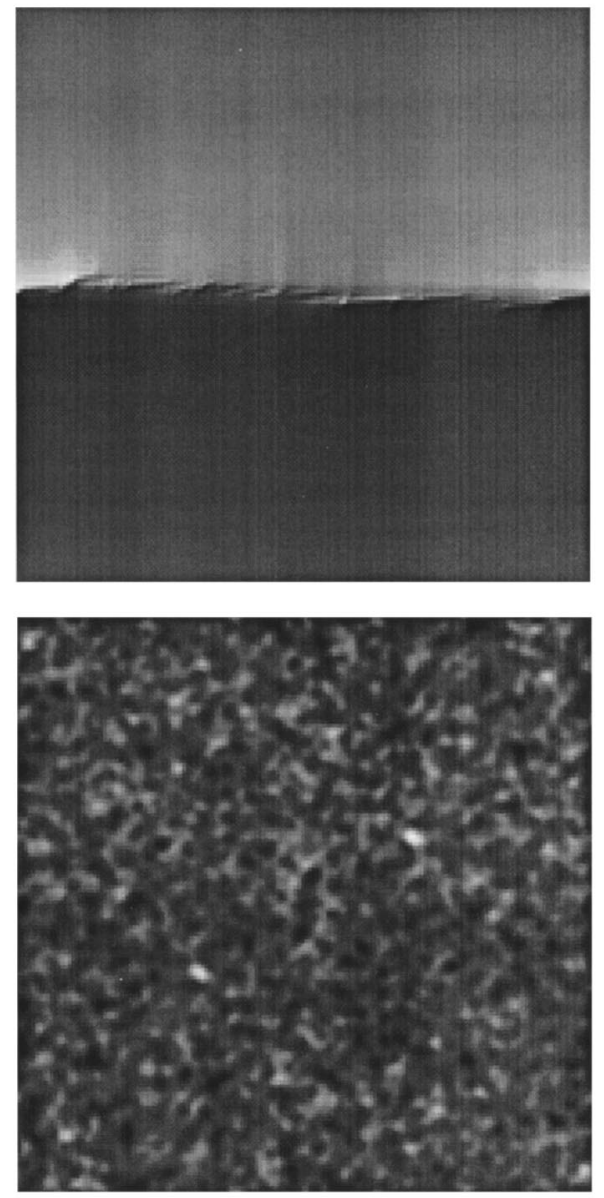

FIG. 7. The Kaiser-Stebbins effect (top) and the full signal, with the Gaussian superposition (bottom) for $\alpha=5$, and $k_{g}=26$. 

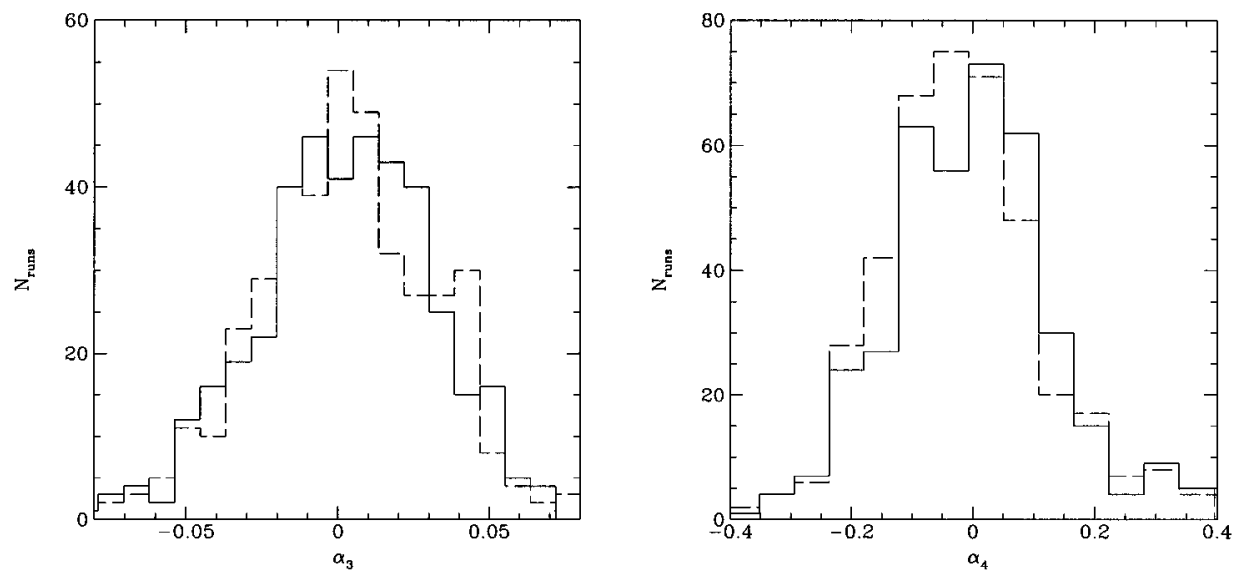

FIG. 8. Histograms of skewness [(a) left] and kurtosis [(b) right] of the gradient of temperature anisotropies from 400 realizations of string maps with Gaussian background (solid lines) and Gaussian realizations with the same power spectrum (dashed lines).

to very distinct linelike discontinuities in the CMB [21,24], the Kaiser-Stebbins effect. In [24] the authors solved Einstein's equations sourced by a high-resolution simulation of an evolving string network. They argued that the nonGaussianity was due to nonrandom phases and illustrated this by generating maps with the same amplitudes but randomized phases and comparing the two. A battery of tests has been used to quantify these non-Gaussian features, in some cases with the inclusion of instrument noise and finite resolution: in [10] the authors looked at gradient histograms and the statistics of the genus of excursion sets, in [13] an analytical fit to the kurtosis of a string map was proposed, and in [23] a multifractal analysis of one-dimensional scans was proposed.

More recent studies of the evolution of string perturbations in the CMB indicate that the Kaiser-Stebbins effect is obscured on subdegree scales by fluctuations generated before recombination [25], and that these perturbations look very Gaussian [26]. None of the previous statistical tests has taken this into account. A careful analysis of the behavior of these two contributions, however, indicates that the nonGaussian features may become dominant again on very small scales: perturbations seeded before recombination will be exponentially suppressed by Silk damping [27] on small scales, while the Kaiser-Stebbins effect will lead to a $k^{-2}$ behavior. This is an ideal situation for using our statistic. We can evaluate the non-Gaussian spectrum on scales where the nonGaussian signal is expected to dominate, and see if it shows any evidence for deviation from the background Gaussian distribution.

If we consider the case of a very small field, we expect to have at most one segment of string crossing the patch. This would be the case for a field of a fraction of a degree. It is instructive to consider the case of a smooth, straight string. Here, the signal is maximally noncircular and all of the power in the ring is concentrated on one of the modes, $\rho_{s}=m_{k} I$, with $\rho_{i}=0$ for $i \neq s$. For such a configuration, the ring spectrum is

$$
\theta_{i}=1 \text { for } i<s, \quad \theta_{i}=0 \text { for } i=s, \quad \theta_{i}=\frac{0}{0} \text { for } i>s .
$$

The last angles are undefined in the same way that the angle $\phi$ in the normal 3D polar coordinates is undefined for points along the $z$ axis. The point remains that the configuration corresponds to a single point on the $\left(m_{k}-1\right)$-dimensional sphere and that, therefore, has probability zero in a Gaussian theory. For display purposes one may then also fix the remaining angles at some particular but arbitrary value. We define $0 / 0=0$.

For a perfectly straight string non-Gaussianity is so extreme that it is visually evident even with a very large amount of background Gaussian noise. The situation changes dramatically, however, for the more realistic case when the string is rough or structured. This is the picture that emerges from high-resolution numerical simulations [28]. The intercommutation of strings will build up kinks and cusps along a string which will only stabilize once gravitational radiation becomes important. Again, most of the power will be concentrated along one or a few modes, leading to a welldefined spectrum up to some maximum $i$. For larger $i$ 's the spectrum will be close to zero or ill defined in the same way as for the straight string case.

Having played with a string code, we have chosen to model the string as a directed Brownian walk along the patch we are considering. We then modeled the effect of the Gaussian background on these scales in the same way as that in the previous example. We superimpose a background Gaussian signal with the power spectrum given in [20]. In Fig. 7(a) we show an example of a $(160)^{2}$-pixel map (20 $\operatorname{arcmin}^{2}$ ) of the non-Gaussian signal and in Fig. 7(b) we superimpose a Gaussian background with a $k_{g}=26$ and with 5 times the overall amplitude of the non-Gaussian signal. Clearly, the beautiful Kaiser-Stebbins effect is now beyond what we can recognize visually. One must, therefore, resort to more abstract tests.

We first applied to our maps some of the standard tests. It has been argued that the skewness and kurtosis of the gradient of the temperature anisotropy field should be a good indicator of string non-Gaussianity. Skewness should be very sharply peaked at zero (the patterns caused by the string are very symmetrical in terms of amplitude), and kurtosis should be larger than that for the Gaussian [13]. In Fig. 8 we show histograms of skewness and kurtosis made from an ensemble of 400 realizations. Clearly, the string with a Gaussian background is indistinguishable from the purely Gaussian sky.

A more elegant statistic involves working out the Euler characteristic of the maps, given a threshold. The procedure is straightforward: given a threshold $\mu \sigma$ one evaluates the 


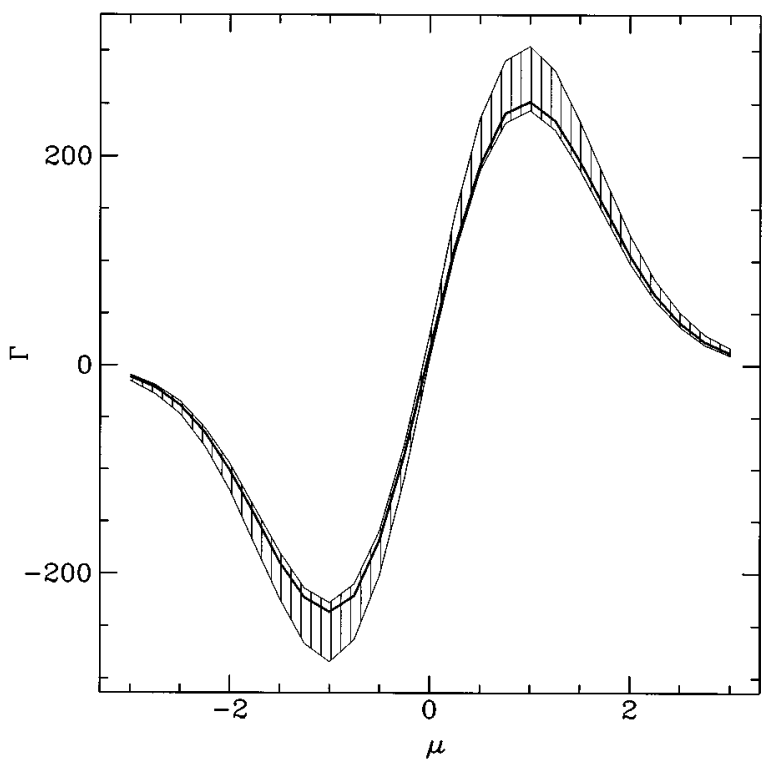

FIG. 9. The mean Euler characteristic $\Gamma$ as a function of threshold for a string map with Gaussian noise (solid line) and for a pure Gaussian map with the same power spectrum (the shaded region is the $1-\sigma$ region around the Gaussian mean, estimated from 100 realizations).

difference between the number of isolated hot regions and cold regions with regard to $t$. For a Gaussian field, the mean genus is

$$
\Gamma \propto \mu e^{-\mu^{2} / 2} .
$$

It was argued in [10] that this would be a good indicator of non-Gaussianity for strings. In Fig. 9 we show the Euler characteristic averaged over 100 runs for the string with a Gaussian background and for a purely Gaussian map with the same power spectrum. Again, we find no significant difference between the two.

Finally, we have applied to these maps our technique. We first looked for the distribution of the $\theta_{i}$ 's in rings where the non-Gaussianity is evident. Due to the random nature of the structure on the string, the signal in the ring spectrum would not be as cleanly defined as that for the straight string case. We, therefore, looked at a large number of maps in order to plot $\theta_{i}$ 's with cosmic or sample invariance error bars. For plotting purposes we shall give error bars as regions of probability larger than $1 / e$. This corresponds to a $1-\sigma$ error bar if the distribution is Gaussian, but generalizes the concept of a 1- $\sigma$ error bar to more general distributions. In particular, the concept may be applied to a uniform distribution, which does not even have a peak. In Fig. 10, the shaded region is where the $\theta_{i}$ 's have more than $1 / e$ probability of being; the ring has $k=70-75$ [for a $(160)^{2}$-pixel map] and we clearly see a ridge towards the left-hand side. For rings at low $k$ this ridge blurs into the standard Gaussian prediction.

A more striking statistic is the inter-ring spectrum. In Fig. 11 , we have shaded the region where $\psi$ 's have more than $1 / e$ probability of being. It is clear that for low values of $k$ the Gaussian background dominates, and the various rings are essentially uncorrelated. However, above a certain threshold, subsequent modes are tightly correlated. As ar-

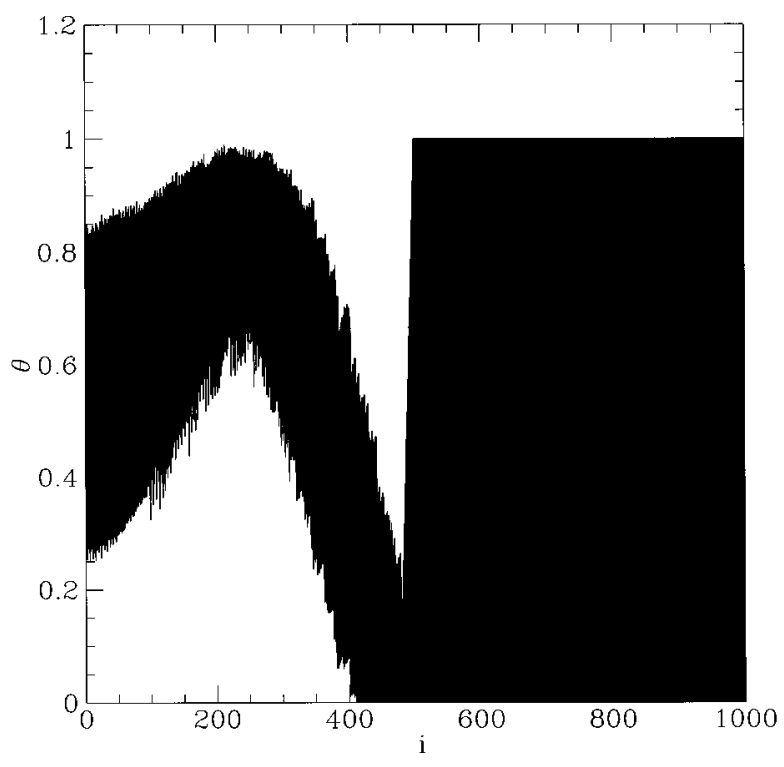

FIG. 10. The ring spectrum for $k=70$ and for $\alpha=5$, and $k_{g}=26$. The shaded region represents a probability larger than $1 / e$ for the values of $\theta_{i}$ to occur.

gued above, most of the power is concentrated along one direction of each ring. What we see here is that this direction is strongly correlated between rings. This quality we labeled as connectedness. We see that strings' connectedness is a robust non-Gaussian feature, even when all else seems to fail.

\section{Evasive non-Gaussian theories}

We finally present a strongly non-Gaussian theory on all scales which evades detection by several traditional nonGaussianity tests. Consider a theory with a power spectrum as in [20], say with $k_{g}=10$, in uncorrelated mesh units. Let

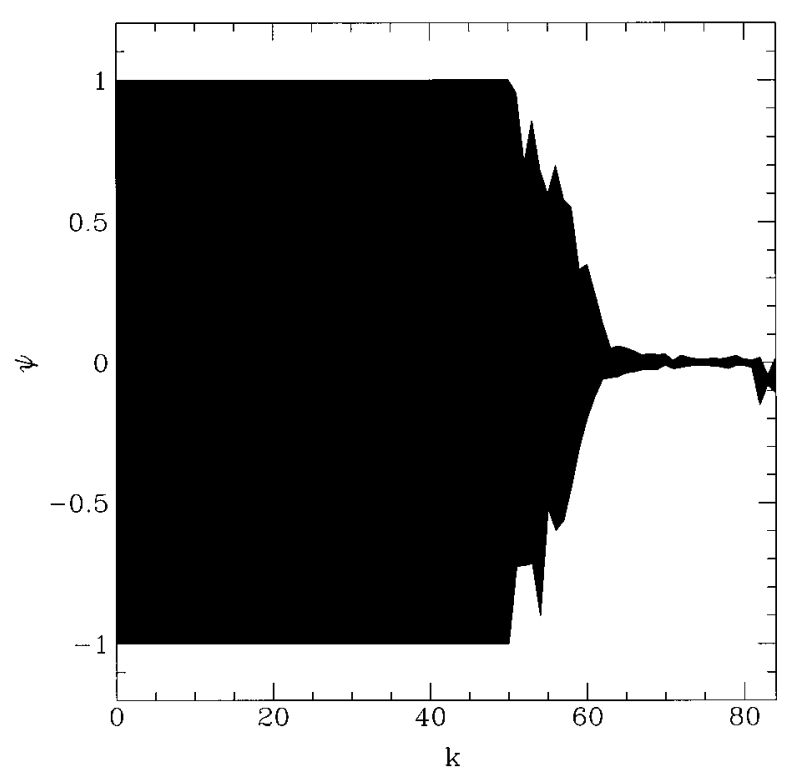

FIG. 11. The inter-ring spectrum with $\alpha=5$, and $k_{g}=26$. The shaded region represents a probability larger than $1 / e$ for the values of $\psi_{i}$ to occur. 

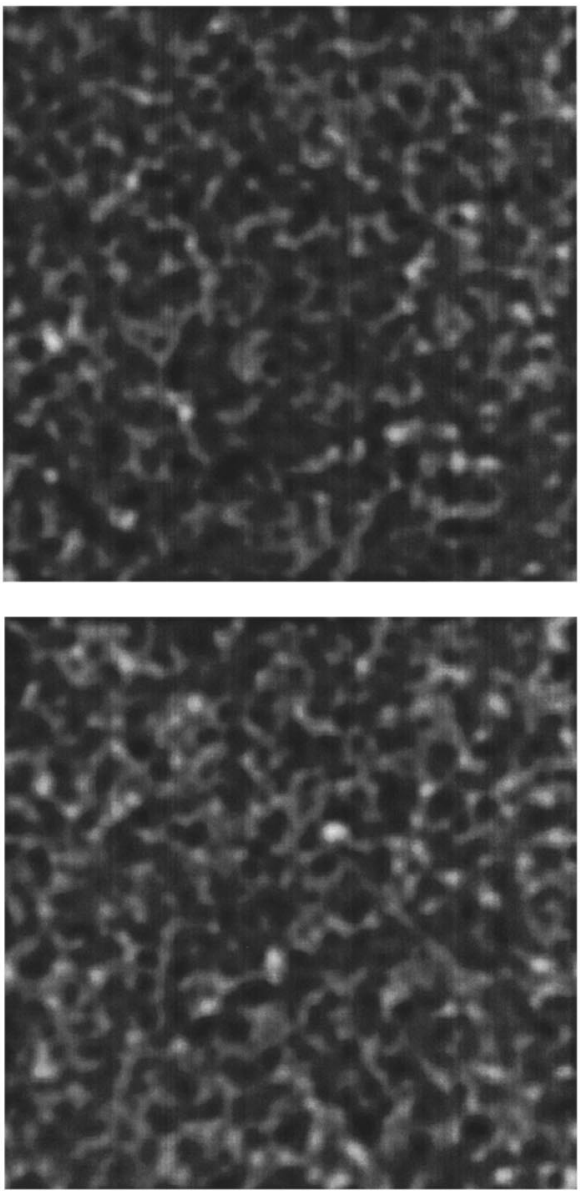

FIG. 12. Realizations of theory $T_{1}$ (top) and $T_{2}$ (bottom). Theory $T_{1}$ is a theory of disconnected, delocalized, perfect spheres, with zero cosmic confusion with theory $T_{2}$, which had the same power spectrum, but is Gaussian.

the phases $\phi$ and inter-ring correlator angles $\psi$ be uniformly distributed. However, let the ring spectra $\theta(k)$ for all rings $k$ be the circular ring spectrum $\theta^{\text {cir }}(k)$ [cf. Eq. (25)] with infinite probability density. Thus, we have theory of delocalized, disconnected spheres. In Fig. 12 we show a realization of this theory (call it theory $T_{1}$ ) and also a Gaussian realization, that is, a realization of a theory (call it $T_{2}$ ) which differs only in that the $\theta(k)$ are now uniformly distributed.

Theory $T_{1}$ is strongly non-Gaussian. The set of all of its realizations has measure zero in any Gaussian theory. In other words, the cosmic confusion between the two theories is zero, where cosmic confusion is defined as the percentage of common skies generated by the two theories [18]. If $Q$ is the set of all map variables, and if $F_{1}(Q)$ and $F_{2}(Q)$ are their distribution functions in the theories $T_{1}$ and $T_{2}$, then the cosmic confusion between the two theories is [18]

$$
\mathcal{C}\left(T_{1}, T_{2}\right)=\int d Q \min \left(F_{1}, F_{2}\right) .
$$

In terms of the variables $Q=\{C(k), \theta(k), \phi, \psi\}$, we have

$$
\begin{gathered}
F_{1}=\prod_{k} \chi_{N_{k}}^{2}(C(k)) \prod_{\phi} \frac{1}{2 \pi} \prod_{\psi} \frac{1}{2 \pi} \prod_{\theta} \delta\left(\theta-\theta^{\mathrm{circ}}\right), \\
F_{2}=\prod_{k} \chi_{N_{k}}^{2}(C(k)) \prod_{\phi} \frac{1}{2 \pi} \prod_{\psi} \frac{1}{2 \pi},
\end{gathered}
$$

so that $\mathcal{C}\left(T_{1}, T_{2}\right)=0$.

Although we have as yet no physical motivation for such a theory, we believe it to be a good example where the traditional beliefs about non-Gaussianity do not hold; in spite of its strong non-Gaussianity this theory evades all tests we have applied to it. Visually, the maps produced by the theory look very Gaussian. We can apply all the tests we have introduced in the previous two sections with rather spectacular failure. Plotting temperature histograms reveals a very Gaussian distribution (see Fig. 13). One may convert these histograms into moments, with the same result. The sections of the $n$-point function which may be computed in practice are also very Gaussian. In Fig. 14 we have plotted the average and 1- $\sigma$ error bars for the collapsed three-point correlation function for $T_{1}$ and $T_{2}$ as inferred from 100 realizations. In Fig. 15 we plot histograms of kurtosis for the two theories. Clearly, they are not good discriminators between the two theories. We can estimate the number of peaks over a given threshold for the two theories. In Fig. 16 we plot the total number of peaks above a given threshold for $T_{1}$ and $T_{2}$. In Fig. 17 we find the Euler characteristic for the two theories. Once again, they are indistinguishable.

Nevertheless, all rings of the $u v$ plane show a ring spectrum which is perfectly circular, without any variance. Any sky, and any $k$, produces a ring spectrum as the one in Fig. 18 , obtained from the same realization used above, for the ring $k=11$.
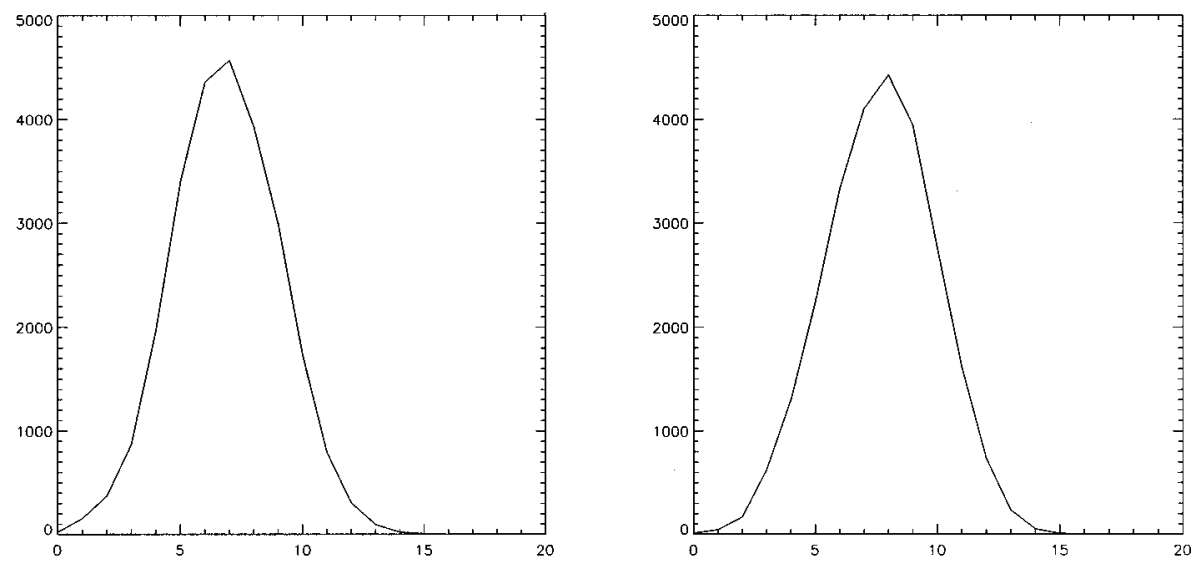

FIG. 13. Temperature histograms for the two maps shown above [that is non-Gaussian (left) and Gaussian (right)]. The skewness is, respectively, 0.043 and -0.068 , and the kurtosis -0.042 and 0.229 . 

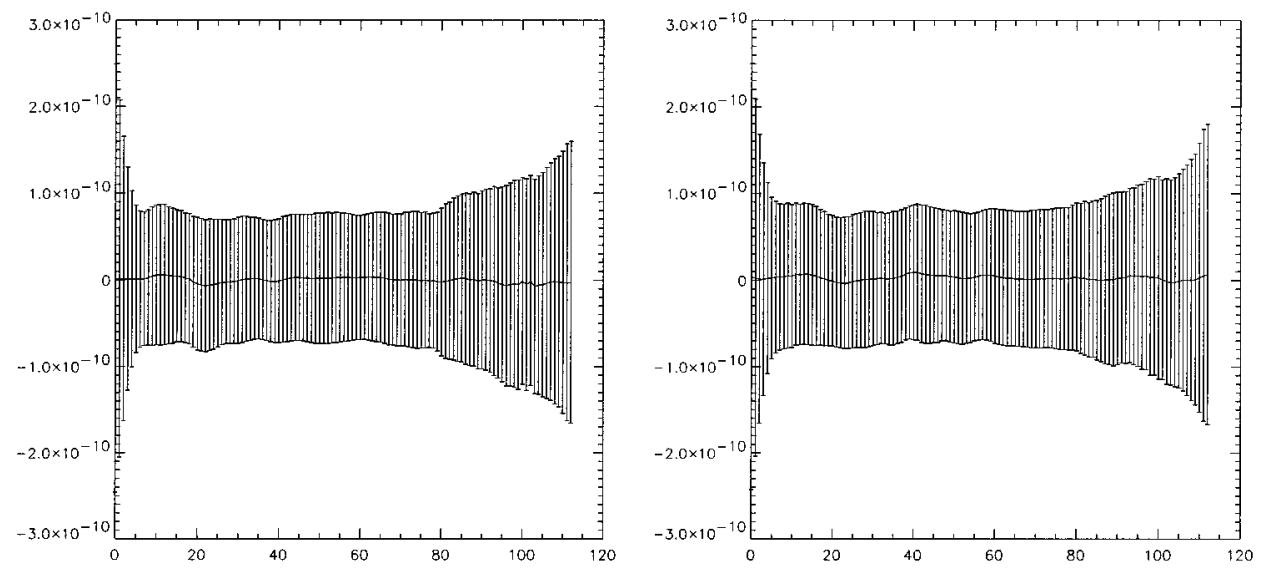

FIG. 14. The collapsed threepoint correlation function for theories $T_{1}$ and $T_{2}$. The averages and error bars were inferred from 100 realizations in both cases.

\section{DISCUSSION}

In this paper we have proposed a transformation of variables in Fourier space which produces non-Gaussian spectra with a particularly simple probability distribution function for a Gaussian random field. We have focused on a subset of these, the ring spectra $\theta_{i}$ and the inter-ring spectrum $\psi$, which contain information about the moduli of the Fourier modes. We have presented a few examples where they are good qualifiers of non-Gaussianity.

A number of comments are in order with regards to the limitations of these statistics. To begin with, these statistics are tailored for data in Fourier space. To actually apply these statistics to real space data will involve nonlocal transformations which may complicate the procedure. However, in the examples which we have worked out, the non-Gaussianity becomes apparent on small scales. Therefore, one is forced to consider experiments with the best possible resolution. These are interferometric devices where the data are measured directly in Fourier space. Another possible shortcoming of these statistics is that they are sensitive to the global shape of the data set or map. This means that if one has

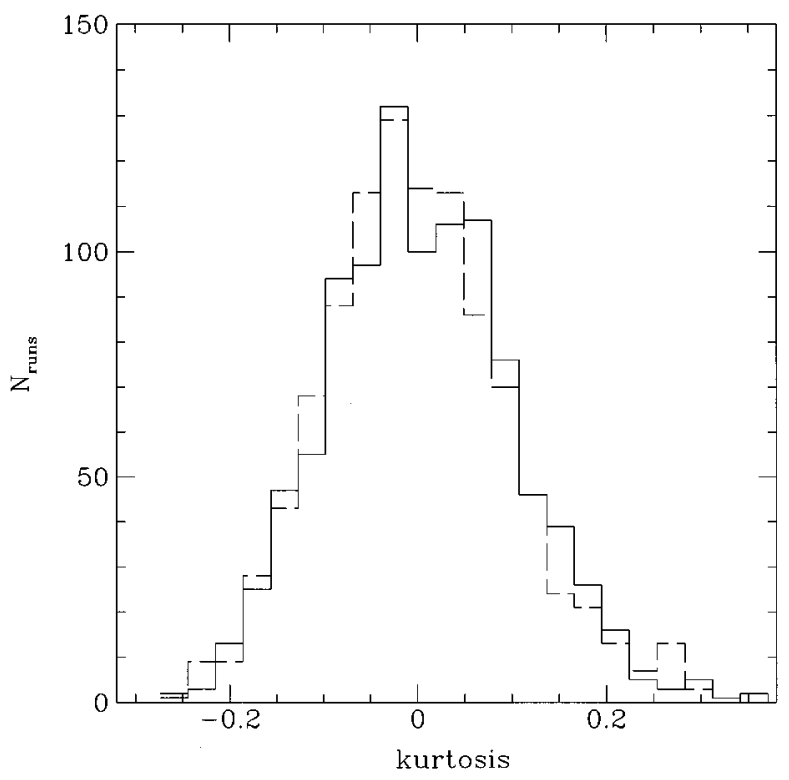

FIG. 15. The histograms of kurtosis for theory $T_{1}$ (solid) and theory $T_{2}$ (dashed) taken from an ensemble of 1000 realizations. many non-Gaussian features (such as many-point sources or many segments of string), then both the ring spectrum and the inter-ring spectrum will look more like Gaussian. This can only be avoided by looking at small fields. But once again this is the situation favored by interferometers. One is limited to small fields (although one can mosaic over reasonably large patches of sky, [29]) and experience in [6] indicates that very few unresolved sources will be present. In an interferometric search for string segments, one would restrict oneself to fields of less than $0.5^{\circ 2}$ and still have a $90 \%$ probability of actually seeing a string, but not more than one.

We have not included the effect of small scale noise in the examples we considered. In those cases the signal was already sufficiently corrupted for it to be difficult to identify the non-Gaussian features. In fact, what one finds is that large scale Gaussianity seems to be more devastating (in terms of erasing non-Gaussian features) than small scale, noise-related, Gaussianity. Clearly, one has to include the two effects if one wants to apply these techniques to data but the details are dependent on each experiment. The statistics defined are nonlinear statistics in the data which means care

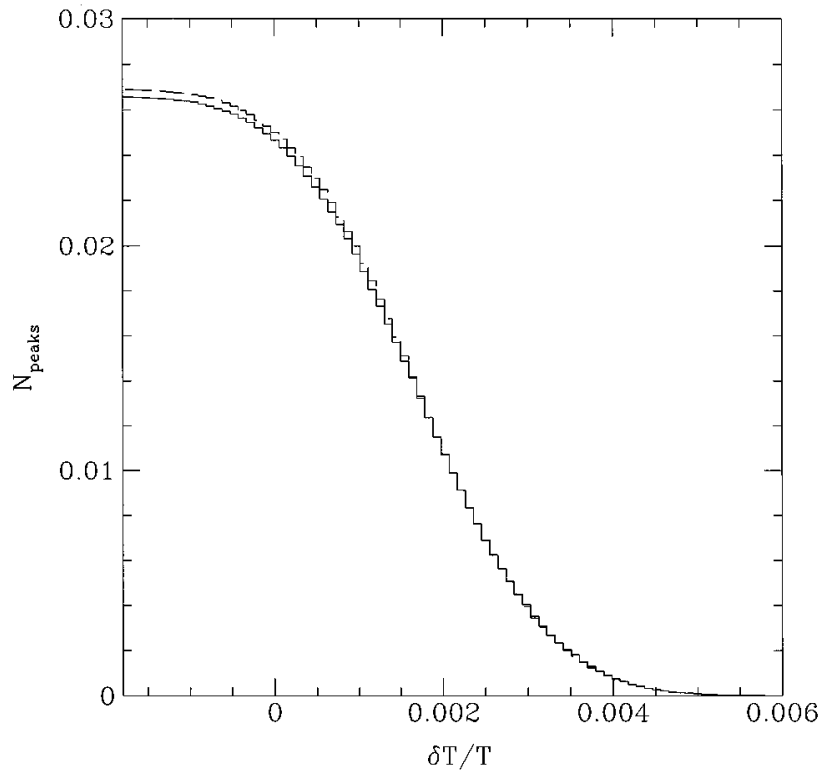

FIG. 16. The density of peaks above a threshold $\delta T / T$ for theory $T_{1}$ (solid line) and theory $T_{2}$ (dashed line). The curves are averaged over 20 runs. 


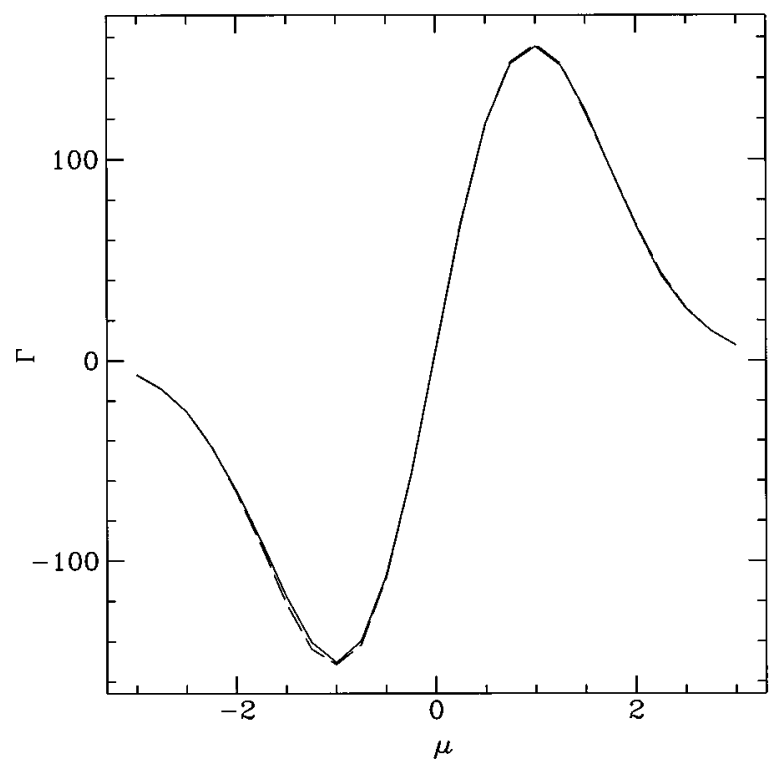

FIG. 17. The average Euler characteristic of theory $T_{1}$ (solid) and $T_{2}$ (dashed), averaged over 100 realizations.

must be taken when considering the effect of noise. A caseby-case analysis of the different observation strategies will have to be made. Again, the fact that the small scale noise in interferometers increases as a power law with scale, as opposed to exponentially as in the case of a single dish experiment, indicates that interferometric devices are the best instruments for testing for non-Gaussian features. One immediate goal will be to design the ideal experiment for detecting the Kaiser-Stebbins effect. This should include a careful analysis of theoretical uncertainties (such as the amplitude of fluctuations at last scattering) as well as the reallife complications mentioned above.

We have focused on statistics with the moduli $\rho$ and have not developed, in any detail, or applied to any example, statistics with the phases $\phi$. It is conceivable that much infor-

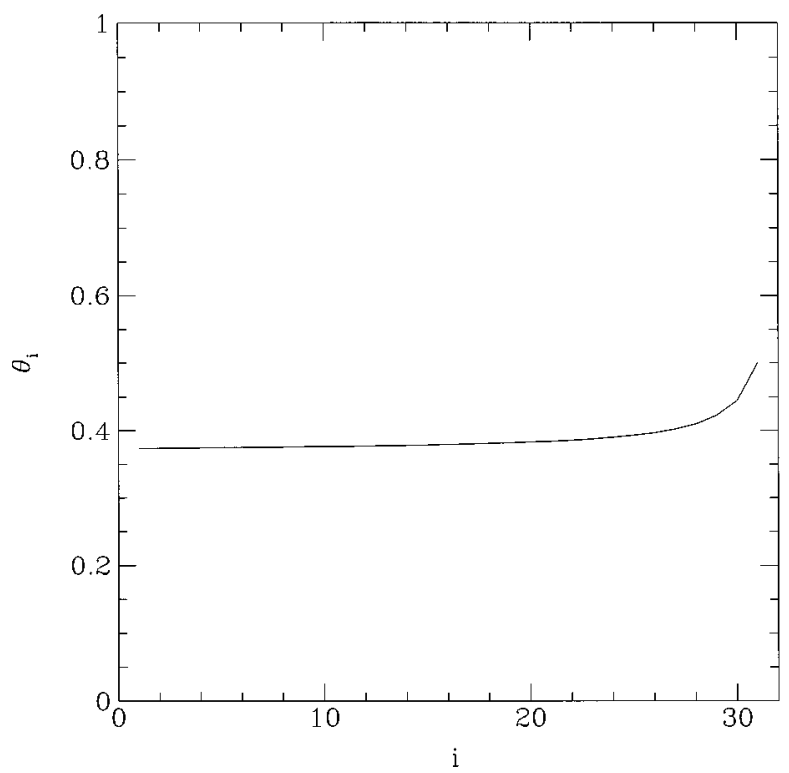

FIG. 18. All rings of theory $T_{1}$ for all realizations show a perfect circular ring spectrum. Here we show the ring $k=11$. mation can be extracted from their behavior. In fact, a generic feature of physically motivated non-Gaussian models is localization, which, as we have argued, is governed by the phases. Although we have organized the information that can be extracted from a finite data set in systematic way, it is important to define a useful set of statistics in terms of the phases. We will do so in [22].

\section{ACKNOWLEDGMENTS}

We thank A. Albrecht, S. Hanany, J. Levin, J. Silk, and L. Tenorio for interesting conversations. P.G.F. was supported by the Center for Particle Astrophysics, a NSF Science and Technology Center at University of California, Berkeley, under Cooperative Agreement No. AST 9120005. J.M. thanks St. John's College, Cambridge, and the Royal Society for support, and also Joe Silk and CfPA for hospitality.

\section{APPENDIX A: INVARIANT SHAPE AND PHASE SPECTRA}

The fact that the uncorrelated mesh points are somewhat undefined makes the search for invariant quantities a pedantic matter. For this reason we decided to define shape variables $\theta$ which, strictly speaking, are not invariant. The interring spectrum, on the other hand, is already invariant.

It is, however, possible to define invariant shapes but they are more complicated. Under a rotation, the moduli $\left\{\rho_{i}\right\}$ suffer a cyclic permutation. Hence, the 2D-rotation group has now become discrete and so it will not discount a degree of freedom. Nevertheless, the angles $\left\{\theta_{i}\right\}$ defined from them will not be invariant under rotations (translations do not affect the $\left\{\rho_{i}\right\}$ ). A way around this is to order the $\left\{\rho_{i}\right\}$ so that the last $\rho_{i}$ is the largest. The angles $\left\{\theta_{i}\right\}$ produced from the ordered $\left\{\rho_{i}\right\}$ will then be properly invariant. They will also always be defined. The joint distribution of the ordered $\left\{\rho_{i}\right\}$ is proportional to the joint distribution of the unordered ones. In fact the Jacobian of any variable interchange is one. One may at most pick a proportionality constant from adding over all the branches of the transformation. Hence, the whole argument in Sec. III still applies, and the new, ordered, $\left\{\theta_{i}\right\}$ will still have a joint distribution which is uniform. However, the new $\left\{\rho_{i}\right\}$ and $\left\{\theta_{i}\right\}$ are now dependent random variables, not because their joint distribution does not factorize, but because the domain of some of the variables depends on those of the others. This results from $\rho_{i} \leqslant \rho_{m}$. This has several unpleasant consequences. For instance, the marginal distribution of any of the $\theta$ 's is now not the factor appearing in the joint distribution function. Hence, the marginal distribution of the properly invariant $\theta$ 's is not uniform, although their joint distribution is. All in all, we found the $\theta$ 's we have defined in the main body of this paper more practical to use, as they are much better behaved in Gaussian theories.

The phases $\phi$ defined in the main body of this paper are also not invariant. Under a rotation they suffer a cyclic permutation, whereas under a translation by a vector $\mathbf{t}$ they transform as $\phi(\mathbf{k}) \rightarrow \phi(\mathbf{k})+\mathbf{k} \cdot \mathbf{t}$. The phases $\phi(\mathbf{k})$ may be seen as an antisymmetric real scalar field on the space $\mathbf{k}$. In this language the field gets rotated under a (real-space) rotation, and acquires a dipole under a (real-space) translation. One can build invariants out of the phases, therefore, simply by subtracting the dipolar component of the field, and aver- 

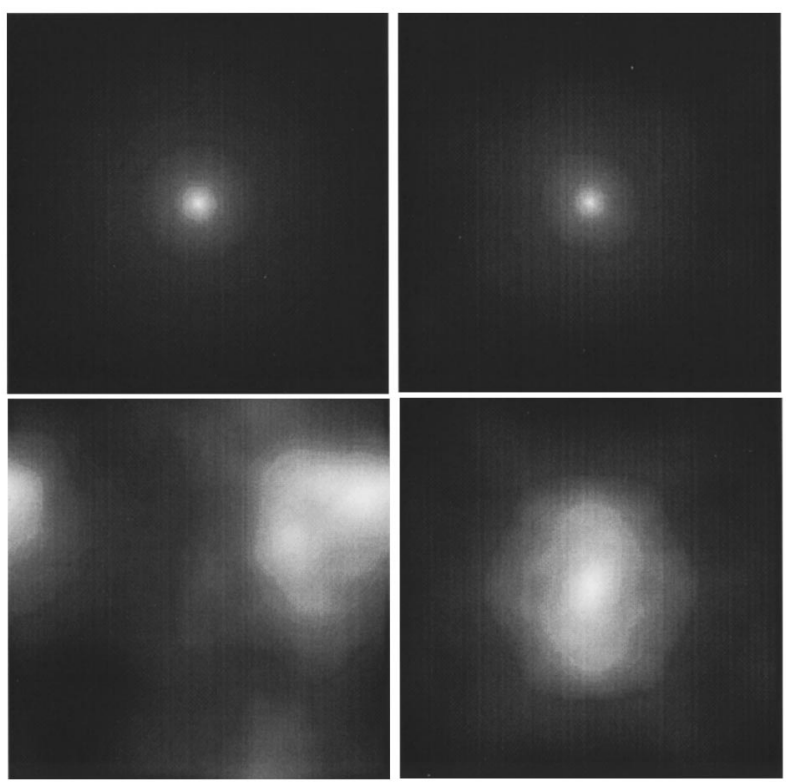

FIG. 19. A spherical hot spot which has been deconstructed at different levels. On the top left-hand panel we have the pure nonGaussian signal. The angles $\theta_{i}$ 's have been redrawn uniformly on the top right picture. On the bottom left the phases $\phi_{i}$ 's were redrawn unformly. On the bottom right, we applied an independent unformly distributed rotation on all rings in Fourier space. From top to bottom and left to right, a plain regular sphere, a shapeless sphere, a delocalized sphere, and a disconnected sphere.

aging over angle. This can be done in many different ways, to be explored more thoroughly in a future publication. Here, we simply outline one possible strategy. Let us in each $\Delta k=1$ ring apply an angular Fourier transform to the phases:

$$
\begin{aligned}
\phi(\mathbf{k})= & \phi\left(k, \beta_{k}\right)=\sum_{m} \phi(k, m) e^{i m \beta_{k}}=\sum_{m} \phi_{c}(k, m) \cos \left(m \beta_{k}\right) \\
& +\phi_{s}(k, m) \sin \left(m \beta_{k}\right) .
\end{aligned}
$$

Then, under a translation, the $m=1$ mode transforms as

$$
\begin{gathered}
\phi_{c}(k, 1) \rightarrow \phi_{c}(k, 1)+k t \cos \beta_{t}, \\
\phi_{s}(k, 1) \rightarrow \phi_{s}(k, 1)+k t \sin \beta_{t},
\end{gathered}
$$

whereas all other modes are invariant. One may then simply throw away the $m=1$ mode, the other ones making up a localization ring spectrum. The distribution of these in Gaussian theories is again not simple, and we shall look for something better than this. This procedure, however, does have the advantage of reacting to individual shapes an localization properties rather than global ones.

For any pair of adjacent $\Delta k=1$ rings we have subtracted two modes too many. These should be returned in the form of two inter-ring phase invariants, such as

$$
\begin{aligned}
& \Phi_{c}(k)=\frac{\phi_{c}(k+1,1)}{k+1}-\frac{\phi_{c}(k, 1)}{k}, \\
& \Phi_{s}(k)=\frac{\phi_{s}(k+1,1)}{k+1}-\frac{\phi_{s}(k, 1)}{k} .
\end{aligned}
$$
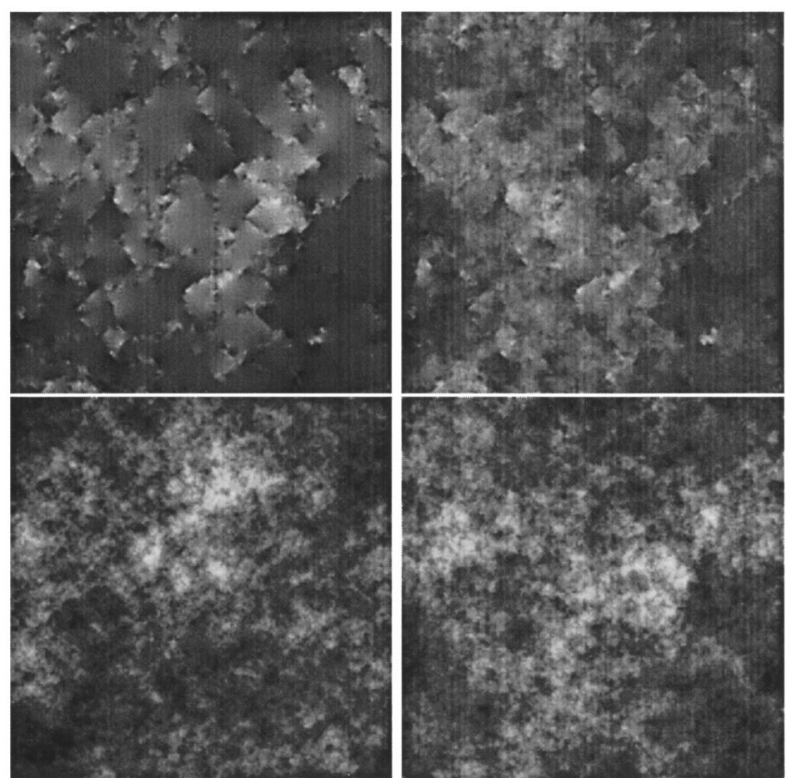

FIG. 20. The Kaiser-Stebbins effect (top left) and its various stages of deconstruction. The angles $\theta_{i}$ 's have been redrawn uniformly on the top right picture. On the bottom left, the phases $\phi_{i}$ 's were redrawn unformly. On the bottom right we applied an independent uniformly distributed rotation on all rings in Fourier space. Respectively, we have strings, shapeless strings, unlocalized strings, and disconnected strings.

Again, this is but one example of a possible invariant made out of phase gradients, to be explored better in our future work.

\section{APPENDIX B: VISUAL INTERPRETATION OF NON-GAUSSIAN SPECTRA}

The decomposition $\{C(k), \theta, \phi, \psi\}$ has an immediate physical interpretation. The angles $\theta$ 's reflect the angular distribution of power and, therefore, reflect shape. The phases $\phi$ transform under translations and so contain the information on position and localization of the structures in the field. The angles $\psi$ 's correlate different scales and, therefore, tell us how connected the structures are. For a Gaussian random field the variables $\{\theta, \phi, \psi\}$ are all uniformly distributed, reflecting complete lack of structure besides the power spectrum. In terms of the various levels of structure considered, we can then characterize Gaussian fluctuations as shapeless, delocalized and disconnected. By comparison with a Gaussian we may then define structure at different levels. We will say that fluctuations, for which $\theta$ 's are not uniformly distributed, are shapeful. If the $\phi$ 's are not uniformly distributed we shall say the fluctuations are localized. If the $\psi$ 's are not uniformly distributed the fluctuations are connected. Although visual structure has room within these definitions, they are considerably more abstract and general. We may consider highly nonvisual types of structure such as shapeful but delocalized fluctuations or disconnected, localized, stringy fluctuations. In this sense we regard our formalism as a robust definition of structure, which goes beyond what is visually recognizable and so is tied down to our particular and narrow path of natural selection. We may imagine an alien civilization with Fourier space eyes (say, interferomet- 
ric eyes [30]), and a brain trained to recognize Fourier space structure at many different levels, structure that would seem totally nonobvious to our human eyes.

To illustrate the limitations of human vision we shall now destroy highly structured maps level by level, that is Gaussianize only one of the variable types $\{\theta, \phi, \psi\}$. Initially, there will be structure at every level, shape, position, and connectedness. We will remove structure gradually, a fact not disasterous for the alien civilization referred above, but which will illustrate the limitations of the human visual method for recognizing non-Gaussianity. In Fig. 19 we play this game with a sphere. We depict a spherical hot spot in real space, then a shapeless sphere, a delocalized sphere, and a disconnected sphere. For the case of a sphere we find that what we recognize as shape is mostly localization. A shapeless sphere keeps its recognizable features. On the other hand, a delocalized sphere loses it characteristic features. Indeed, the idea of a shapeful but nonlocalized object sounds somewhat surreal for all we can visually conceptualize. Nev- ertheless, our formalism will reveal the strong but not obvious non-Gaussianity exhibited by a delocalized sphere.

In Fig. 20 we repeat the same exercise for a map displaying the Kaiser-Stebbins effect from cosmic strings. Shapeless strings, delocalized strings, and disconnected strings are shown. Considerable disarray is introduced in every case, but one may say that disconnected strings as well as delocalized strings are perhaps the most messy of them. This is consistent with the strong signal in $\psi$ we have found for the case of the realistic Kaiser-Stebbins effect. On the other hand, the fact that linelike discontinuities are present even for shapeless strings shows how much more structure there is in the map on top of the structure which we can recognize. This is important since the beautiful patchwork is very fragile to the hard realities of noise and superposed Gaussian signal. In the real world, it turns out, the nonvisual feature, which is the connectedness of strings, happens to survive much better than the patchwork (which reflects mostly localization).
[1] P.J.E. Peebles, Principles of Physical Cosmology (Princeton University Press, Princeton, NJ, 1993).

[2] P. Steinhardt, in Particle and Nuclear Astrophysics and Cosmology in the Next Millenium, Snowmass 94, Proceedings of the Summer Study, Snowmass, Colorado, edited by E. Kolb and R. Peccei (World Scientific, Singapore, 1995), Report No. astro-ph/9502024 (unpublished).

[3] Kogut et al., Report No. astro-ph 9601062 (unpublished).

[4] S. Landy et al., Astrophys. J. 456, L1 (1996).

[5] A. Vilenkin and P. Shellard, Cosmic Strings and other Topological Defects (Cambridge University Press, Cambridge, England, 1994); T.W.B. Kibble, J. Phys. A 9, 1387 (1976).

[6] M. Hobson, in Proceedings of the Moriond conference on CMB, 1996 (unpublished).

[7] J.R. Bond and G. Efstathiou, Mon. Not. R. Astron. Soc. 226, 655 (1987).

[8] N. Vittorio and R. Juskiewicz, Astrophys. J. 314, L29 (1987).

[9] P. Coles, Mon. Not. R. Astron. Soc. 234, 509 (1988).

[10] J.R. Gott et al., Astrophys. J. 352, 1 (1990).

[11] A. Gangui and S. Mollerach, Phys. Rev. D 54, 4750 (1996).

[12] R. Scaramella and N. Vittorio, Mon. Not. R. Astron. Soc. 263, L17 (1993).

[13] R. Moessner, L. Perivolaropoulos, and R. Brandenberger, Astrophys. J. 425, 365 (1994).

[14] D. Coulson, P. Ferreira, P. Graham, and N. Turok, Nature (London) 368, 27 (1994).

[15] U. Pen, D. Spergel, and N. Turok, Phys. Rev. D 49, 692 (1994).

[16] P.J.E. Peebles, The Large Scale Structure of the Universe
(Princeton University Press, Princeton, NJ, 1980).

[17] R. Adler, The Geometry of Random Fields (Wiley, New York, 1981).

[18] J.C.R Magueijo, Phys. Lett. B 342, 32 (1995).

[19] M. Hobson and J. Magueijo, Mon. Not. R. Astron. Soc. (to be published).

[20] G. Efstathiou, in Physics of the Early Universe, edited by J. Peacock, A. Heaven, and A. Davis (Scottish University Summer School Publications, Bristol, England, 1990), pp. 361470.

[21] N. Kaiser and A. Stebbins, Nature (London) 310, 391 (1984).

[22] P.G. Ferreira and J. Magueijo (in preparation).

[23] M.P. Pompilio, F.R. Bouchet, G. Murante, and A. Provenzale, Astrophys. J. 449, 1 (1995).

[24] F.R. Bouchet, D.P. Bennet, and A. Stebbins, Nature (London) 335, 410 (1988).

[25] A. Albrecht, D. Coulson, P. Ferreira, and J. Magueijo, Phys. Rev. Lett. 76, 1413 (1996); J. Magueijo, A. Albrecht, D. Coulson, and P. Ferreira, ibid. 76, 2617 (1996); J. Magueijo, A. Albrecht, P. Ferreira, and D. Coulson, Phys. Rev. D 54, 3727 (1996).

[26] N. Turok, Report No. astro-ph/9606087 (unpublished).

[27] J. Silk, Astrophys. J. 151, 459 (1968).

[28] D.P. Bennett, in Formation and Evolution of Cosmic Strings, edited by G. Gibbons, S. Hawking, and T. Vachaspati (Cambridge University Press, Cambridge, England, 1990); F. R. Bouchet, ibid.; E.P.S. Shellard and B. Allen, ibid.

[29] J. Carlstrom, M. White, and P.G. Ferreira (in preparation).

[30] After all we have Fourier space ears. We thank Dr. K. Baskerville for this pertinent remark. 